\title{
CENTRALITY OF THE UMBILICAL CORD INSERTION IN A HUMAN PLACENTA INFLUENCES THE PLACENTAL EFFICIENCY
}

\author{
Michael Yampolsky ${ }^{1}$, Carolyn M. Salafia ${ }^{2,3}$, Oleksandr Shlakhter ${ }^{4}$, Danielle Haas ${ }^{5}$, Barbara \\ Eucker $^{6}$, and John Thorp 6 \\ ${ }^{1}$ Department of Mathematics, University of Toronto, 40 St. George St, Toronto, Ontario, Canada, \\ M5S2E4 \\ 2 Placental Analytics, LLC, Larchmont, NY, USA \\ ${ }^{3}$ Department of Obstetrics and Gynecology and Pediatrics, New York Methodist Hospital, Brooklyn, \\ NY \\ ${ }^{4}$ Department of Mechanical and Industrial Engineering, University of Toronto, 5 King's College \\ Road, Toronto, Ontario, Canada M5S3G8 \\ ${ }^{5}$ Department of Pathology, St Luke's Roosevelt Hospital, NY, NY, 10019 \\ ${ }^{6}$ Department of Obstetrics and Gynecology, University of North Carolina at Chapel Hill, Chapel Hill, \\ NC 27599
}

\begin{abstract}
Goal: We assess the effect on placental efficiency of the non-centrality of the umbilical cord insertion and on chorionic vascular distribution to determine if cord centrality measurably affects placental function as reflected in birth weight.
\end{abstract}

Materials and Methods: 1,225 placentas collected from a prospective cohort had digital photographs of the chorionic plate. Of these, 1023 were term, 44 had velamentous cord insertion and 12 had missing clinical data, leaving $\mathrm{N}=967(94.5 \%)$ cases for analysis. Mathematical tools included a dynamical stochastic growth model of placental vasculature, Fourier analysis of radial parameterization of placental perimeters, and relative chorionic vascular density (a measure of "gaps" in the vascular coverage) derived from manual tracings of the fetal chorionic surface images. Bivariate correlations used Pearson's or Spearman's rank correlation as appropriate, with $\mathrm{p}<0.05$ considered significant.

Results: The correlation of the standard deviation of the placental radius (a measure of nonroundness of the placenta) with cord displacement was negligible ( $r=0.01)$. Empirical simulations of the vascular growth model with cord displacement showed no deviation from a normal round-tooval placental shape for cord displacement of $10-50 \%$ of placental radius. The correlation of the metabolic scaling exponent $\beta$ with cord displacement measured by Fourier analysis is 0.17 ( $p<$ 0.001). Analysis of the chorionic vascular density in traced images shows a high correlation of the

(C) 2009 Elsevier Ltd. All rights reserved.

Corresponding author: Michael Yampolsky, PhD Department of Mathematics University of Toronto 40 St. George Street, Toronto, Canada M5S2E4 yampolsky.michael@gmail.com.

Publisher's Disclaimer: This is a PDF file of an unedited manuscript that has been accepted for publication. As a service to our customers we are providing this early version of the manuscript. The manuscript will undergo copyediting, typesetting, and review of the resulting proof before it is published in its final citable form. Please note that during the production process errors may be discovered which could affect the content, and all legal disclaimers that apply to the journal pertain. 
relative vascular distance with cord displacement: 0.59 in one set of 12 images, and 0.20 in the other set of 28 images.

Conclusion: Non-central cord insertion has little measurable correlation with placental shape in observed or simulated placentas. However, placentas with a displaced cord show a markedly reduced transport efficiency, reflected in a larger value of $\beta$ and hence in a smaller birth weight for a given placental weight. Placentas with a non-central cord insertion have a sparser chorionic vascular distribution, as measured by the relative vascular distance. Even if typically a placenta with a noncentral insertion is of a normal round shape, its vasculature is less metabolically effective. These findings demonstrate another method by which altered placental structure may affect the fetal environment, influencing birth weight and potentially contributing to later health risks.

\section{INTRODUCTION}

A given placental weight can produce clinically normal newborns with a wide range of birth weights. Thus fetal growth is the product of more than the simple accumulation of placental size. How might a given placenta size yield different birth weights? Parental genetic contributions may have effects on the constitutionally appropriate birth weight, and potentially on placental weight as well. Maternal weight gain, medical illness, environmental exposures and habits such as tobacco use can further modify fetal as well as placental growth. Genetics apart, we have shown that placental proportions, such as deviations of the placental shape from round, and the relative thickness of the placenta, modify placental functional efficiency, and, in particular, explain some of the range of birth weights possible in a normal population delivered from similar placental weights $[1,2,3]$ and account for some of the variance in birth weight that otherwise has been attributed to effects of maternal factors such as age, parity, race/ ethnicity and socioeconomic status [1].

In $[2,3]$ we have argued that the mechanism by which the placental shape is related to the placental efficiency is its relation to the structure of the underlying placental vascular tree. The placental vascular tree is the fetus' sole source of oxygen and nutrients. Recent studies suggest that, at a population level, fetal oxygenation parallels birth weight [4], another indicator that fetal growth is inextricably linked to fetal oxygenation. The chorionic plate vessels form a high capacitance/ low resistance blood distribution system that moves large volumes of blood quickly from the umbilical cord to the placental villi, the sites of nutrient and oxygen exchange, and back to the umbilical cord. Alterations in the basic proportions of the placental disk [2] as well as the structure of the vascular tree may decrease the optimality of the transport efficiency, and thus the efficacy by which placental mass functions to yield fetal mass [3].

In this paper we study the effect on the placental efficiency of the non-centrality of the umbilical cord insertion. We show that eccentricity of cord insertion is associated with a sparser chorionic vascular distribution, and, ultimately, with a reduced transport efficiency of the placental vasculature. The latter results in a reduced birth weight for a given placental weight. Velamentous and even marginal cord insertion has been, in the words of K. Benirschke and P. Kaufmann, "moderately well correlated with small placentas and fetuses" [6]. We hypothesize that associations of these extreme insertions to poor fetal outcomes are not limited only to extreme insertions. While extreme marginal and membranous cords are specifically more mechanically vulnerable to torsion or compression than cords inserted on the cushion of the chorionic plate, our focus is more on what biological process(es) are that lead to such extreme cord insertions, assigning some pathologic connotations to even non-marginal but markedly eccentric cords.

"Trophotropism" has been considered the "force" that underlies eccentricity (or non-centrality) of the umbilical cord insertion, and is generally thought of in terms of variability in the permissiveness of the maternal environment to uniform placental growth. In this theory, a less 
"permissive" or more variable intrauterine environment is the cause of asymmetry of placental growth about the umbilical cord insertion [7]. We feel "trophotropism" may be thought of more generally, in terms of specific endocrine or growth factor environments that alter normal placental branching growth, rather than as a simple variability in local uterine "receptivity". Regardless of the term used, we hypothesize that any factor that modifies the uniformity of placental branching growth results in the development of a deformed placenta and one that is less functionally efficient [2,3]. Such deformation may be manifested in asymmetry of placental growth about the umbilical cord. In this context, we predict that the more eccentric the umbilical cord insertion, the less efficient the placenta in terms of the birth weight per gram of placental weight.

\section{MATERIALS AND METHODS}

\section{Placental Cohort}

The Pregnancy, Infection, and Nutrition Study is a cohort study of pregnant women recruited at mid pregnancy from an academic health center in central North Carolina. Our study population and recruitment techniques are described in detail elsewhere [8]. Beginning in March 2002, all women recruited into the Pregnancy, Infection, and Nutrition Study were requested to consent to a detailed placental examination. As of October 1, 2004, 94.6 percent of women consented to such examination. Of those women who consented, 87 percent had placentas collected and photographed for image analysis. Of the 1,225 consecutive placentas collected, 1023 were delivered at term. Velamentous cord insertion is well recognized as abnormal [6]. Such cords are, in particular, more mechanically vulnerable to torsion or compression, than cords inserted on the chorionic plate. To avoid biasing our findings by the lower birth weights associated with velamentous cords, we have excluded the 44 cases in which the cord was not inserted on the chorionic plate. Further 12 cases were excluded because of missing data. This left $\mathrm{N}=967$ cases for analysis, 94.5 percent of all the placentas delivered at term in our sample. Placental gross examinations, histology review, and image analyses were performed at EarlyPath Clinical and Research Diagnostics, a New York State-licensed histopathology facility under the direct supervision of Dr. Salafia. The institutional review board from the University of North Carolina at Chapel Hill approved this protocol.

The fetal surface of the placenta was wiped dry and placed on a clean surface after which the extraplacental membranes and umbilical cord were trimmed from the placenta. The fetal surface was photographed with the Lab ID number and $3 \mathrm{~cm}$. of a plastic ruler in the field of view using a standard high-resolution digital camera (minimum image size 2.3 megapixels). A trained observer captured series of $x, y$ coordinates that marked the site of the umbilical cord insertion, the perimeter of the fetal surface, and the "vascular end points", the sites at which the chorionic vessels disappeared from the fetal surface. The perimeter coordinates were captured at intervals of between $1 \mathrm{~cm}$ and $2 \mathrm{~cm}$, and more coordinates were captured if it appeared essential to accurately capturing the shape of the fetal surface.

\section{Software}

Numerical simulations were carried out using DLA-placenta, a Unix-based, ANSI C, software package developed under the terms of the GNU General Public License as published by Free Software Foundation. For visualizations we have used PovRay: a freeware ray tracing program available for a variety of computer platforms; and Maplesoft Maple 11.0 Math \& Engineering software.

\section{Quantifying the centrality of the umbilical cord insertion and roundness of placental shape}

As the first approach to calculating the centrality of the umbilical cord insertion, we calculated the center of mass of the perimeter markers of the chorionic plate surface. We then calculated 
the cord displacement as the planar distance between the center of mass and the umbilical insertion point. The relative cord displacement is the same quantity divided by the placental diameter.

Another measure of the centrality of the cord is obtained as follows. First, the umbilical insertion point is placed at the origin. Perimeter markers are connected by straight line segments to obtain an approximate perimeter $P$ of the chorionic plate. A sector of opening of $6^{\circ}$ with vertex at the origin is rotated in $6^{\circ}$ increments. For each turn of the sector, the points in $P$ inside of it are averaged to yield a radial marker. In this way, we obtain 60 radii emanating from the origin spaced at $6^{\circ}$ intervals. They are connected to obtain the angular radius $\mathrm{r}(\theta)$, which is a function of the angle $\theta$ from the umbilical insertion point. The function $r(\theta)$ can be analyzed using the standard techniques of Fourier series. In particular, we computed the first Fourier coefficient of $r(\theta)$ as:

$$
C=\frac{1}{\pi} \int_{0}^{2 \pi} e^{-t \theta} r(\theta) d \theta
$$

The quantity $|\mathrm{C}|$ can be used as a measure of the centrality of the umbilical cord. We will refer to it as Fourier displacement. It measures the "average oscillation" of the placental radius in one full turn around the umbilical insertion point. For a perfectly round placenta Fourier displacement is equal to the distance between the umbilical insertion point and the geometrical center (Fig. 1).

We have used a measure of roundness of a placenta which is calculated as follows. The geometric center of the chorionic plate is calculated by averaging the points on the perimeter. Then, in a $18^{\circ}$ sector emanating from the geometric center, a mean radial distance to the perimeter is calculated. The sector is rotated in $18^{\circ}$ increments to produce 20 sectorial radial measurements. We then calculate the mean square deviation of these measurements. We denote this quantity $\sigma$, and call it deviation from roundness. For a round placenta, regardless of the centrality of the umbilical insertion, $\sigma=0$. A large value of $\sigma$ indicates a very non-round placental shape.

\section{Modeling vascular trees of placentas}

We have described a model of placental angiogenesis in [2]. The model is based on a random fractal growth process known as Diffusion Limited Aggregation, or DLA. The key step in the process is identifying the tips of the vascular tree which sprout new growth at a given moment of time. This is achieved by noting where a diffusion particle released at a large distance from the tree is likely to land. In such a formulation, the probability of a new growth in a fragment of the tree is what mathematicians call the harmonic measure of the fragment. If the tree were made of a charged conductor, and a unit charge was placed on it, then the harmonic measure of a fragment of the tree is equal to its total charge. According to the well-known principle of electrostatics, the charge will be concentrated at the tips of the tree, where the new growth will occur. Further details and parameters of the model are described in [2].

\section{Measuring the vascular density of the placenta, real and model}

In the digital photographs of the placentas of our dataset the large vascular branches on the chorionic surface were traced by a trained and experienced observer (D.H.) Then, for each pixel in the chorionic surface, the minimal distance to a traced vessel was calculated. To normalize to the size of the image, this quantity was then divided by the square root of the area of the placental image calculated in pixels. The resulting number is a dimensionless measure which we call the relative chorionic vascular distance $\Delta$ of a placenta. A lower value of the 
relative vascular distance means a better penetration of the chorionic surface by the blood vessels (smaller gaps between the main vascular branches in the placental surface).

A calculation of the relative chorionic vascular distance was also carried out for model placental vascular trees. In this case, the tree is represented as a digitized collection of pixels of various size, with the larger pixels corresponding to large vascular branches. Removing the smaller pixels leaves only the large vessels. After this, the calculation of $\Delta$ proceeds as above.

\section{Measuring the effectiveness of the placenta as a transport network}

We recall that in $[3,10]$ we have demonstrated that a baby's birth weight (BW) normally scales with the placental weight (PW) at delivery as

$$
P W \approx B W^{0.75} .
$$

As a consequence, the quantity

$$
\beta={ }^{\log P W} / /_{\log B W}
$$

measures how efficient the placenta is in nourishing the fetus: a larger value of $\beta$ means a less efficient placenta [3]. As explained in [3], the value of $\beta$ is a biologically relevant version of the fractal dimension of the placental vasculature. In [2], a higher value of $\beta$ is related to a deformed placental vascular tree. More specifically, it indicates a suppressed placental vascular growth.

A related measure of the placental effectiveness is the corrected fetal-placental ratio [3]:

$$
F P R_{\text {corrected }}=B W^{0.75} / P W \text {. }
$$

As seen in $[3,10]$, the normal value of this measurement is close to 1 , and a lower value indicates a less effective placenta, one less efficient in terms of its ability (per gram of placental weight) to transfer nutrients to the fetus to permit fetal growth and birth weight.

\section{RESULTS}

\section{Centrality of the insertion has little effect on the placental shape}

As we have verified in [9] the normal placental shape is round, with a central umbilical insertion. Numerical simulations of the placental vasculature using our DLA model of placental angiogenesis [2] were carried out with a displaced umbilical cord insertion. This is achieved by placing the initial seed of the model asymmetrically with respect to the direction of the growth. Simulations were carried out in 20 instances, with cord displacement varying from $10 \%$ to $50 \%$ of the placental radius. In all of the simulations there was no significant deviation from a normal round-to-oval shape (Figure 2). Thus, the empirical model indicates that the shape of the chorionic plate of a placenta is not significantly affected by the centrality of the umbilical cord insertion.

To test this hypothesis, we have used the deviation from roundness $\sigma$ as the quantitative measure of non-roundness of a placenta. In confirmation of our assumption the correlation of the value of $\sigma$ with the cord displacement variable in our dataset is only 0.01 . Thus, non-centrality of the cord insertion produces little measurable effect on the roundness of the shape. Below, we will 
argue that (somewhat surprisingly) it has a significant effect on the underlying vascular structure of the placenta, and thereby on its metabolic effectiveness.

\section{Cord centrality and clinical characteristics}

We have found no association of either the cord displacement or the Fourier displacement with specific maternal diseases, parity, gender of the baby, habitus, weight gain in pregnancy, or smoking. Maternal age has a significant positive correlation with both measures of noncentrality of the insertion (Table 1): with cord displacement the correlation is $0.086(\mathrm{p}=0.007)$ and with Fourier displacement the correlation is $0.097(\mathrm{p}=0.002)$.

\section{Cord centrality significantly impacts placental efficiency: non-central cord insertion for the same placental weight results in a smaller baby}

We note first, that placentas with larger value of the cord displacement tend to be larger in size. The value of the cord displacement in the dataset did not show a statistically significant correlation with the mean placental radius (correlation 0.046, $\mathrm{p}=0.152$ ) and was significantly correlated with the placental weight (correlation $0.138, \mathrm{p}<0.001$ ). To determine if the placentas with a large cord displacement were as metabolically efficient as the normal ones, we have calculated the correlation of cord displacement with the scaling exponent $\beta={ }^{\log P W} / /_{\log B W}$. It is large $(0.133)$ and very significant $(\mathrm{p}<0.001)$. When we use the Fourier displacement $|\mathrm{C}|$ as the measure of the cord displacement, the correlation with $\beta$ is even larger (correlation 0.17, $\mathrm{p}<0.001)$. On the other hand, the cord displacement is not significantly correlated with the birth weight (see Table 1).

Thus, the placentas with a large umbilical cord displacement, measured either as a distance from the geometric center, or as $|\mathrm{C}|$, are less metabolically efficient. These placentas grow heavier than normal, and the birth weight of the baby is not significantly affected. Thus the added placental weight does not fully translate into the corresponding gain for the birth weight. Placentas with a non-centrally inserted cord tend to produce smaller babies than normal placentas of the same weight.

\section{Cord centrality and the vascular density}

Both real and model placentas with an asymmetrically inserted umbilical cord are typically of a normal, round to oval, shape. However, we will argue that the structure of the underlying vascular tree of such a placenta is deformed. As a quantitative measure of this we have used the relative chorionic vascular density $\Delta$, as described in the Methods.

We first investigated the impact of non-central insertion on the value of $\Delta$ in model placentas. Models were chosen both with a symmetrically placed umbilical cord, and with an asymmetric cord placement. We have found that in a model placenta, a cord displacement of $20-25 \%$ of the placental diameter results in an increase in the relative chorionic vascular distance of at least $20 \%$. Thus, the model suggests that a round placenta with an asymmetrically placed umbilical cord has a significantly lower vascular coverage of the chorionic surface. This suggests, that an asymmetric umbilical insertion will result in a lower vascular coverage, and hence a reduced metabolic efficiency of the placenta.

We have then selected 40 traced placental images from our dataset with a round shape. These were further divided into two groups, according to the resolution with which the digital photographs were taken: the first of 12 placentas, and the second of 28 placentas. The first group contains placentas of a more uniform round shape, with the values of the cord displacement between 1.2 and $5 \mathrm{~cm}$. The correlation of the cord displacement with the relative chorionic vascular distance in this sample is 0.59 . The largest value of the displacement corresponds to the largest value of $\Delta$, which is some $50 \%$ above the mean. Further, the four 
placentas with the larges displacements $(4-5 \mathrm{~cm})$ in this sample also correspond to the four largest values of $\Delta$. In the second group of 28 placentas, the shapes were less uniformly round, and the effect of the cord displacement on $\Delta$ is less obviously pronounced. Still the correlation of the cord displacement with $\Delta$ in this group is 0.2 , and the largest value of $\Delta$ (again more than $50 \%$ above the mean) corresponds to the fourth largest cord displacement $(5 \mathrm{~cm})$.

Thus we again find that larger values of cord displacement correspond to altered chorionic surface shapes, and by extension, altered placental vascular trees, with less metabolically effective structure (see Figure 3).

\section{DISCUSSION}

We have demonstrated that a non-central insertion of the umbilical cord is a source of deformation of the macroscopic placental vascular architecture that can be captured in digital images of the chorionic surface vasculature. This is somewhat unexpected, as the shape of a placenta with a non-central insertion remains round, as confirmed both by our statistical analysis, and by the dynamical model of placental growth we have previously presented in [2]. Even if typically a placenta with a non-central insertion is of a normal round shape, it is heavier (and thus, thicker), its surface vascular distribution is sparse but less metabolically effective, as reflected by $\beta$ larger. This results in a smaller birth weight for the same placental weight.

The altered structure of the surface vasculature can be seen from our measurements of the coverage of the placental surface with the large branches of the vascular tree. The placentas with a non-centrally inserted cord suffer from a sparser vascular coverage, so that a point on the surface is typically further away from a large blood vessel than in a normal placenta. But as we have argued previously in [3], the easiest-to-grasp indicator of the deformation of the placental vascular architecture as a whole (both macroscopic and microscopic finer structure of placental, stem and terminal villi) is the metabolic scaling exponent $\beta$ calculated as the ratio of the logarithms of the placental weight and the baby birth weight. The quantity $1 / \beta$ should be seen as a biologically relevant version of the fractal dimension of placental vasculature, so the larger value of $\beta$ implies a poorer placental functional efficiency and, as we speculate, an altered placental vascular fractal. We have shown that the value of $\beta$ is strongly and significantly correlated with non-centrality of the cord insertion.

Placentas with a non-centrally inserted umbilical cord tend to be heavier. Their diameters are not significantly affected, and thus they are thicker than normal. We can speculate that the increased thickness is a compensatory mechanism for a reduced efficiency per unit of placental weight.

Abnormalities of cord centrality are not specific to any maternal pathology, which suggests that it is due to a deviation in a key early developmental placental process. As maternal age (which is commonly correlated with paternal age) increases, the likelihood of a displaced cord grows. This suggests that the magnitude of the deviation is amplified in an aged conceptus or imposed on the conceptus by an aging maternal intrauterine environment.

Various authors have studied the placental transport efficiency in utero by the umbilical venous blood flow measurement using color/pulsed Doppler ultrasound (e.g. [11-14]) or 3D power Doppler measurements of the placental blood flow (e.g. [15-19]), and have noted the effect that different placental attachments in utero have on the transport efficiency. It would be a promising future direction of study to correlate our measurements (the scaling exponent $\beta$, the measures of cord displacement, and the chorionic vascular density) with the Doppler data obtained in utero. As noted in [3], for clinical applications, measuring the placental efficiency 
by calculating the value of the scaling exponent $\beta$ has the advantage of simplicity: only BW and PW are needed, so it can be assessed instantly after birth.

We present two measures of the non-centrality of the umbilical cord insertion. The simpler one is the distance between the geometric center of the placental surface and the umbilical insertion point. The other measure, which we denote $|C|$, is obtained by a simple Fourier analysis of the placental perimeter. Computation of these measures from a digital photograph of a placenta is easily automated. The latter measure appears to be more effective than the former in quantifying the non-centrality. In particular, it gives a much better correlation with the change in $\beta$. Fourier analysis is a natural tool for the study of two-dimensional placental shapes, and in a forthcoming work we plan to further develop this approach and apply these methods to the study of placental growth in maternal and fetal disease states. The computation of the chorionic vascular density $\Delta$ is not entirely automated at present, as it requires tracing of the surface vasculature by a highly trained observer. Let us mention a project [20] in this regard, which, we hope, will replace the human observer by a computer.

Current standards for describing umbilical cord insertion site have not changed since the recommendations of Kurt Benisrchke in 1961 [21]. The umbilical cord insertion, when actually measured in centimeters, is measured to the nearest disc edge, and is not considered in relation to either the larger or smaller placental diameters. The umbilical cord insertion may also be merely described qualitatively as central, eccentric, marginal, or velamentous. The present work extends our prior demonstrations that irregular placental shapes are associated with reduced birth weight for given placental weight -- using both the relatively crude descriptions of "round/oval versus all other shapes" in the Collaborative Perinatal Project [5], or using the digital photographs that were the materials of the current study [22]. This present work suggests that clinical placental diagnostics should also include a careful measure of umbilical cord insertion site as an important indicator of placental functional integrity and as also an easily recorded measure of chorionic vascular deformation, which is more difficult at present to quantify. Obtaining a simple digital photograph of the fetal surface of the placenta is readily accomplishable in most surgical pathology departments, in which gross photographs may be routinely obtained to document features of, for example, excised cancers.

\section{Acknowledgments}

This work was partially supported by NSERC Discovery Grant (M. Yampolsky), by NARSAD Young Investigator Award (C. Salafia), by K23 MidCareer Development Award NIMH K23MH06785 (C. Salafia).

\section{References}

1. Salafia CM, Zhang J, Charles AK, Bresnahan M, Shrout P, Sun W, Maas EM. Placental characteristics and birthweight. Paediatr Perinat Epidemiol May;2008 22(3):229-39. [PubMed: 18426518]

2. Yampolsky M, Salafia C, Shlakhter O, Haas D, Eucker B, Thorp J. Modeling the variability of shapes of a human placenta. Placenta 2008;29:790-797. [PubMed: 18674815]

3. Salafia C, Yampolsky M. Metabolic scaling law for fetus and placenta. Placenta 2009;30:468-471. [PubMed: 19285342]

4. Lackman F, Capewell V, Gagnon R, Richardson B. Fetal umbilical cord oxygen values and birth to placental weight ratio in relation to size at birth. Am J Obstet Gynecol 2001;185(3):674-82. [PubMed: 11568797]

5. Salafia CM, Zhang J, Miller RK, Charles AK, Shrout P, Sun W. Placental growth patterns affect birth weight for given placental weight. Birth Defects Res A Clin Mol Teratol 2007;79(4):281-8. [PubMed: 17286292]

6. Benirschke, K.; Kaufmann, P. Pathology of the Human Placenta. Vol. 4th ed.. Springer; NY: 2000. p. 947 
7. Kraus, FT.; Redline, RW.; Gersell, DJ.; DM, Nelson; JM, Dicke. Disorders of Placental Development. Armed Forces Institute of Pathology, American Registry of Pathology; Washington DC: 2004. Placental Pathology, Atlas of nontumor Pathology, First Series, Fascicle 1; p. 57Chapter 4

8. Savitz DA, Dole N, Williams J, et al. Determinants of participation in an epidemiological study of preterm delivery. Paediatr Perinat Epidemiol 1999;13:114-25. [PubMed: 9987790]

9. Yampolsky M, Shlakhter O, Salafia C, Haas D. Mean surface shape of a human placenta. e-print Arxiv.org, 0807.2995

10. Salafia D, Misra M, Yampolsky A, Charles R, Miller. Allometric metabolic scaling and fetal and placental weight. Placenta 2009;30:355-360. [PubMed: 19264357]

11. Figueras F, Fernándes S, Hernándes-Andrade E, Grataćs E. Umbilical venous blood flow measurement: accuracy and Reproducibility. Ultrasound Obstet Gynecol 2008;32:587-591. [PubMed: 18618412]

12. Boito S, Struijk PC, Ursem NTC, Stijnen Th. Wladimiroff JW. Umbilical venous volume flow in the normally developing and growth-restricted human fetus. Ultrasound Obstet Gynecol 2002;19:344349. [PubMed: 11952962]

13. Barbera A, Galan HL, Ferrazzi E, Rigano S, Józwik M, Battaglia FC, Pardi G. Relationship of umbilical vein blood flow to growth parameters in the human fetus. Am J Obstet Gynecol 1999;181:1149-1153. [PubMed: 10561635]

14. Galan HL, Jozwik M, Rigano S, Regnault TRH, Hobbins JC, Battaglia FC, Ferrazzi E. Umbilical vein blood flow determination in the ovine fetus: Comparison of Doppler ultrasonographic and steady-state diffusion techniques. Am J Obstet Gynecol 1999;181:174-179. [PubMed: 10411816]

15. Yu C-H, Chang C-H, Ko H-C, Chen W-C, Chang F-M. Assessment of Placental Fractional Moving Blood Volume Using Quantitative Three-dimensional Power Doppler Ultrasound. Ultrasound Med Biol 2003;29:19-23. [PubMed: 12604113]

16. Mercé LT, Barco MJ, Bau S. Reproducibility of the study of placental vascularization by threedimensional power Doppler. J Perinat Med 2004;32:228-233. [PubMed: 15188796]

17. Mercé LT, Barco MJ, Bau S, Kupešic S, Kurjak A. Assessment of Placental Vascularization by Threedimensional Power Doppler "Vascular Biopsy" in Normal Pregnancies. Croatia Med J 2005;46:765771.

18. Guiot C, Gaglioti P, Oborto M, Piccoli E, Rosato R, Todros T. Is three-dimensional power Doppler ultrasound useful in the assessment of placental perfusion in normal and growth-restricted pregnancies? Ultrasound Obstet Gynecol 2008;31:171-176. [PubMed: 18254148]

19. Noguchi J, Hata K, Tanaka H, Hata T. Placental Vascular Sonobiopsy Using Three-dimensional Power Doppler Ultrasound in Normal and Growth Restricted Fetuses. Placenta 2009;30:391-397. [PubMed: 19327824]

20. Almoussa, N.; Dutra, B.; Lampe, B.; Getreuer, P.; Wittman, T.; Salafia, C.; Vese, L. Automated Vasculature Extraction from Placenta Images; Abstract submitted to SPIE Medical Imaging Conference; San Diego. 2010.

21. Benirschke, K. Examination of the placenta. Prepared for the Collaborative Study on Cerebral Palsy, Mental retardation and other Neurological and Sensory Disorders of Infancy and Childhood, National Institute of Neurological Diseases and Blindness, US Department of Health, Education and Welfare, Public Health Service; 1961.

22. Salafia CM, Maas E, Thorp JM, Eucker B, Pezzullo JC, Savitz DA. Measures of placental growth in relation to birth weight and gestational age. Am J Epidemiol Nov 15;2005 162(10):991-8. [PubMed: 16192346] 


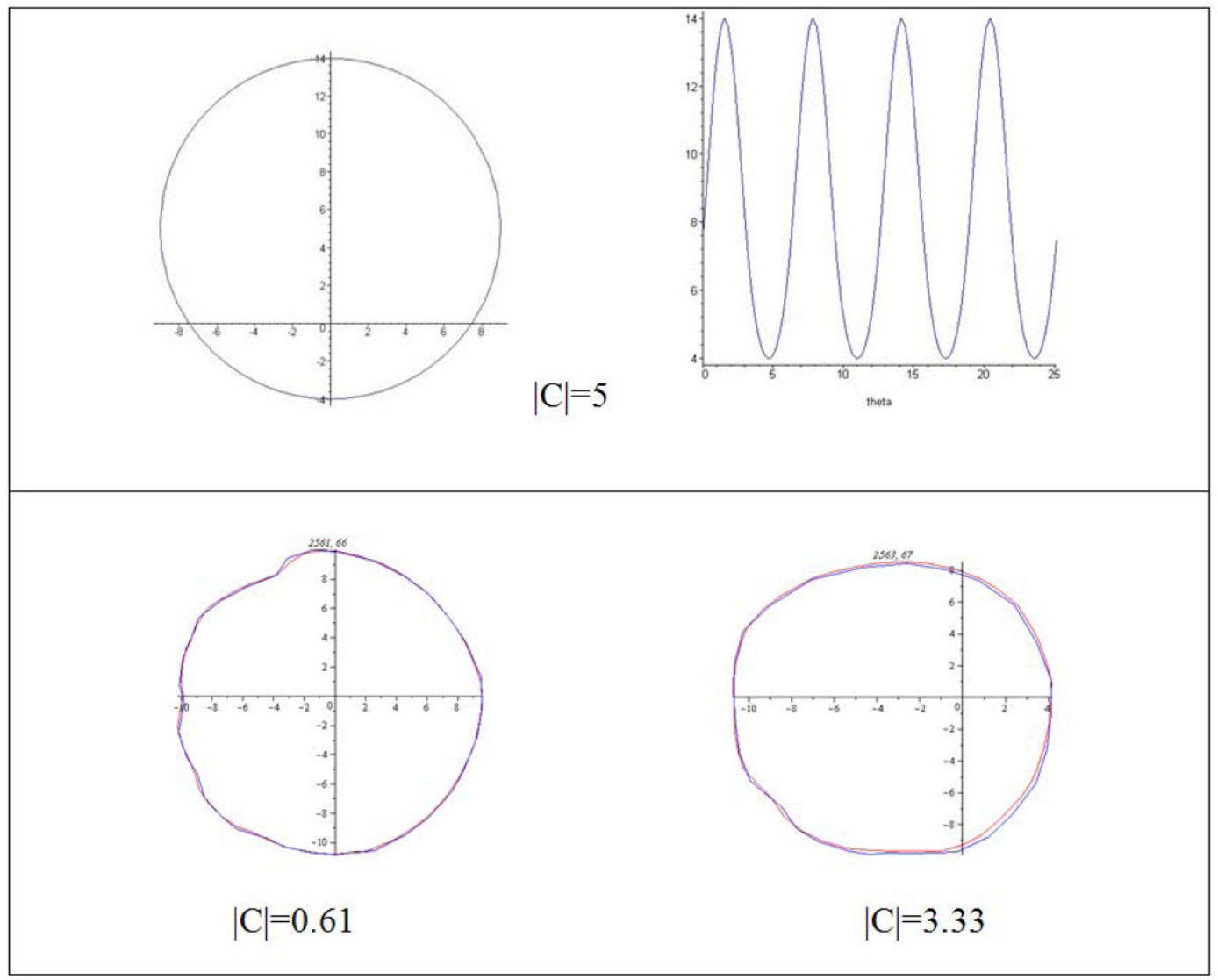

Figure 1. 


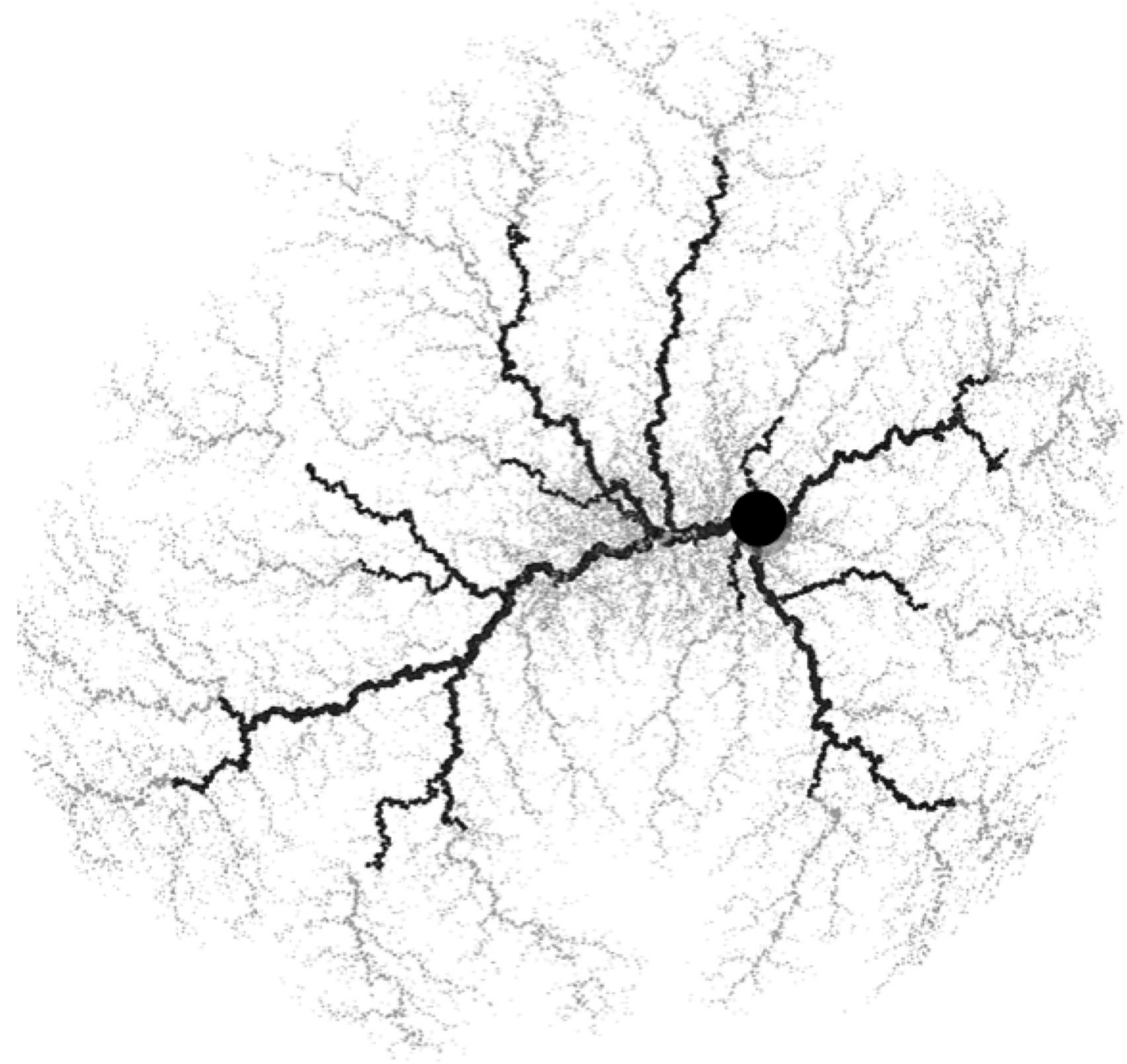

Figure 2. 


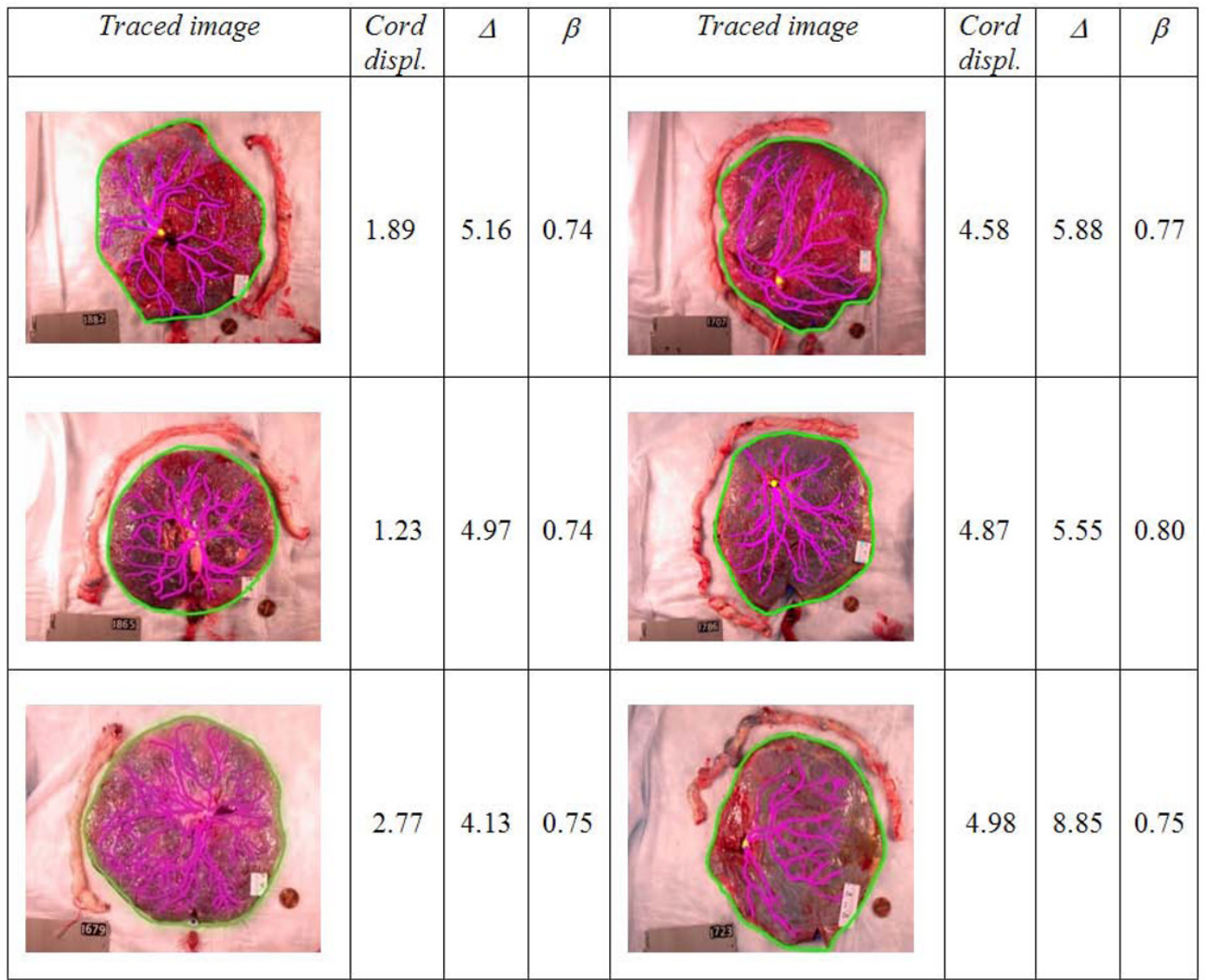

Figure 3. 


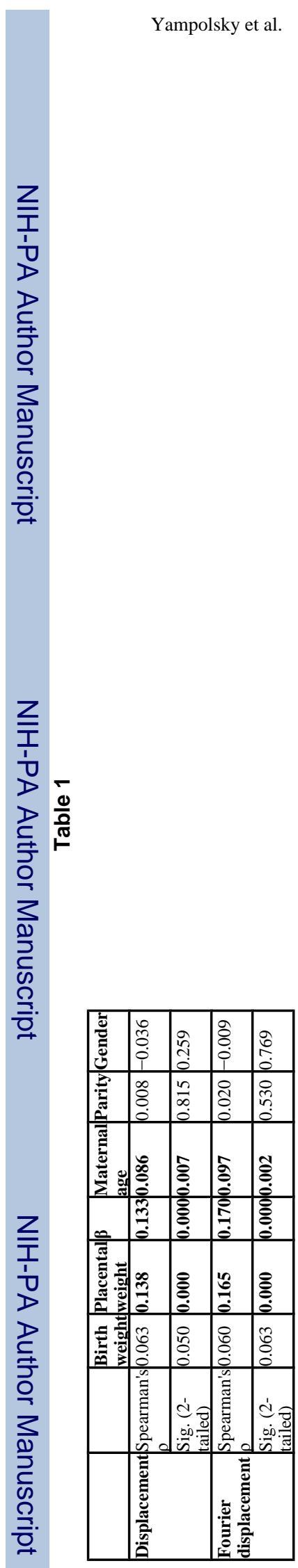

Page 13

Placenta. Author manuscript; available in PMC 2010 December 1. 